\title{
Modified Simulated Annealing Algorithm (MSAA) for DC-DC Converters
}

\author{
D.O.I - 10.51201/12522 \\ https://doi.org/10.51201/12522
}

\author{
B. S. Sudame*1 \\ S. G. Kadwane ${ }^{2}$ \\ R.S.Somalwar ${ }^{3}$ \\ ${ }^{* 1}$ Department of Electrical Engineering, Yeshwantrao Chavan College of Engineering Nagpur, India. \\ ${ }^{* 2}$ Department of Electrical Engineering, Yeshwantrao Chavan College of Engineering Nagpur, India. \\ ${ }^{3}$ Department of Electrical Engineering, Bajaj Institute of Technology, Wardha ,India
}

\begin{abstract}
Performance of Neural Network based Model Reference Adaptive Controller is influenced by the requirement of a plant Emulator or evaluation of plant derivatives. This paper addresses a Simulated Annealing (SA) based MSAA algorithm to improve the response without evaluating the exact plant derivatives or constructing a plant Emulator for buck-converter.
\end{abstract}

Keywords: Feed forward neural networks; Robustness; Model reference adaptive control; simulated annealing.

\section{Introduction}

The criteria of global stability in procedure of design and applying corresponding adaptive control law can be achieved through introduction of elementary idea of MRAC i.e. Model reference Adaptive Control. Aim to apply such system is to fulfil the requirement of stability criterion. But the system constructed on MRAC requires a plant Emulator or the prior knowledge of plant derivatives, which is difficult to obtain in case of nonlinear systems. The nonlinearity present in dynamical system can be perfectly handled by Neural Networks. Current prevalent research suggests that systems especially neuro control direct type work efficiently in this [3]-[5] to avoid and surpass these problems. In [8], [12]-[13] Park et al, for computing the derivation associated with the system neuro identifier concept is suggested. In [6] Suwat et al, proposed offline trained Neural Network based plant emulator. In either case the display of actions of the controller is dependent on the plant modeling, which is difficult for non-linear plants.

In this paper for MRAC, a training of neutral network which is feed forward using MSAA algorithm is proposed. The algorithm is capable of handling any nonlinear system and is free from Emulator and plant derivatives also the training algorithm gives guaranteed stability. The other advantage using this algorithm is that it avoids the Local minima problem associated with general backpropagation algorithm. The simulation study for a DC-to-DC converter (Buck type) is presented. The various results show the improved performance. 


\section{The Simulated Annealing (SA) algorithm for the training of Neural}

\section{Network}

The SA algorithm follows the process in which it determines the nethermost energy of pulverized state of physical systems with several interrelated atoms. The utmost competent way of finding a global least possible is shown by a scalar cost function [9].

The annealing processes provides for further development. Seldom, we can use with less cost effective methods too. This can minimize the frequency of getting obstructed at the local minimum. The optimization property of the SA can be suitably used to find the optimal weight configuration of the neural controller for the MRAC based system as shown in Fig 1.

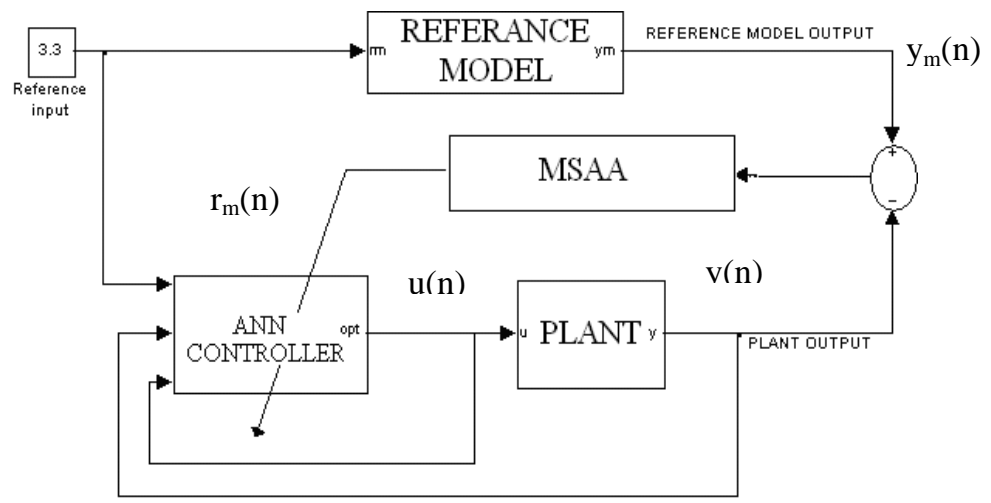

Fig 1: Basic structure of SA trained MRAC

Labelling of the tablet "REFERENCE MODEL" in Fig 1 is the essential route of the plant to be followed. The objective is to find a control action law, $u(n)$, for all $n>0$, and an apprising law for controller parameters such that .

$$
\lim _{n \rightarrow \infty}\left|y_{m}(n)-y(n)\right| \leq \varepsilon
$$

For a specified constant $\varepsilon>0$.

For a Neural system in Fig 2, the weight updating law using simulated annealing can be formulated as given below.

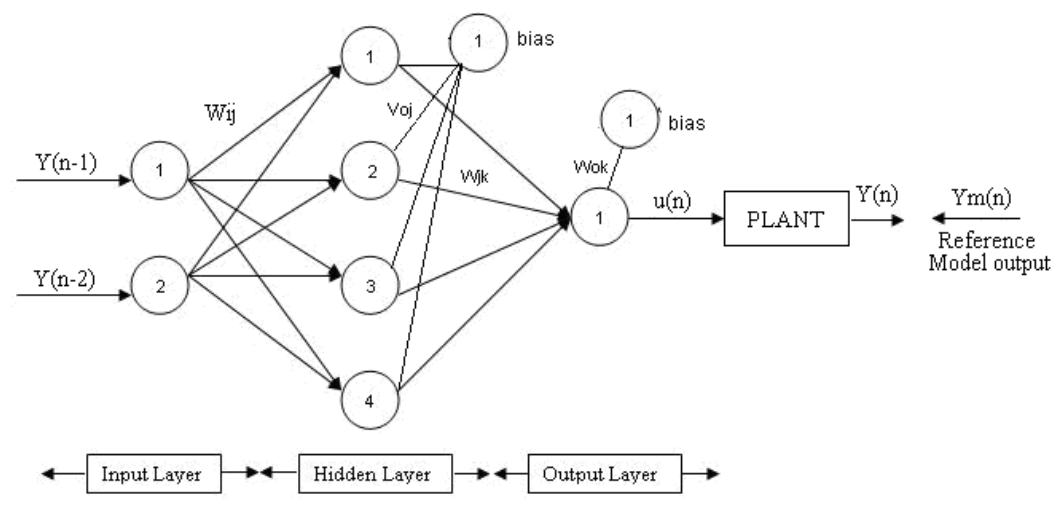

Fig 2: Basic structure of Neural Controller 
If $x_{i}(n)$ represents the input vector, $w_{i j}(n), w_{j k}(n)$ represents the input to hidden and hidden to output weight vectors, $y(n)$ and $y_{m}(n)$ represents the plant output and reference model output respectively. The cost function can be written as.

$$
J=\frac{1}{2}\left(y_{m}(n)-y(n)\right)^{2}
$$

The adjustable parameters of the neural systems are its weight vectors $\mathrm{w}_{\mathrm{ij}}, \mathrm{w}_{\mathrm{jk}}$. SA algorithm searches the optimal weight configuration in heuristic fashion with equation (2). If the $J$ is minimized from its previous iteration we keep the current configuration else it is accepted by a probability $\left(\Delta e(n) / T K_{b}\right)$, where $\Delta e$ is the difference between the cost functions of the two weight configurations. The algorithm can be formulated as below.

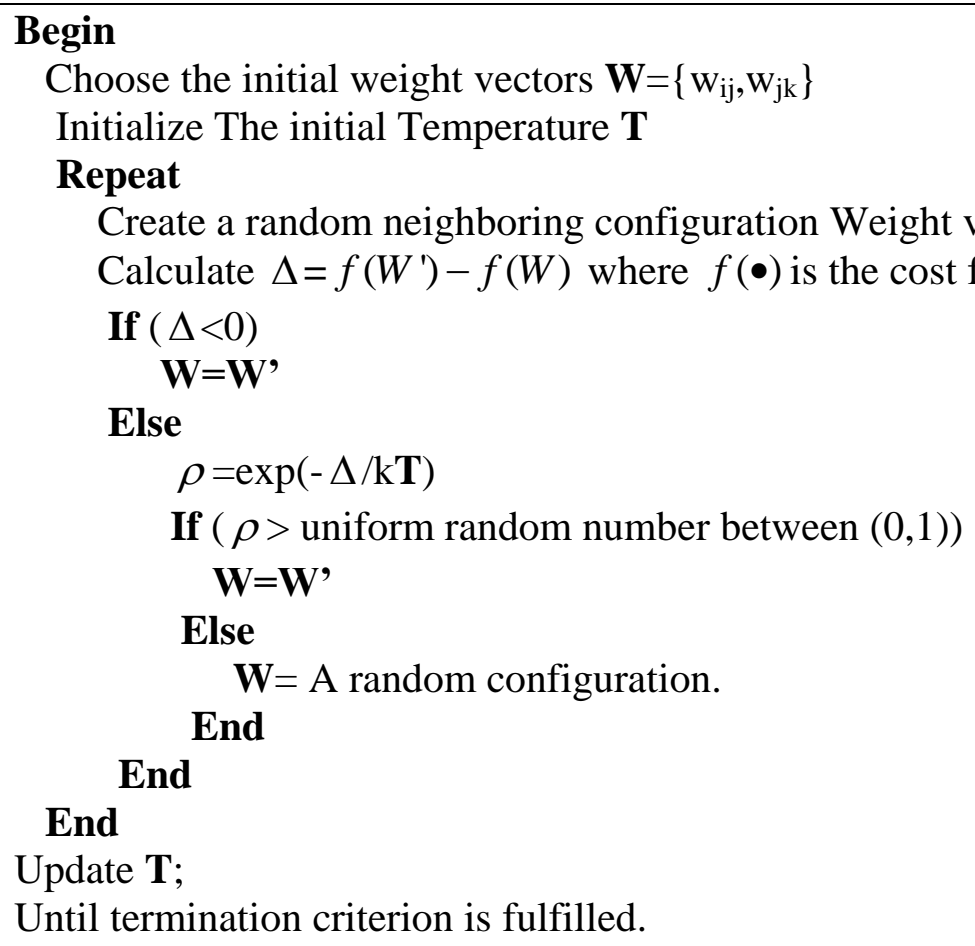

End

Table 1: The conventional SA procedure for the Feed Forward Neural Network MRAC $\mathrm{T}$ is the temperature parameter of the SA process $K_{b}$ is the Boltzman constant. For the simulation purpose the 4-7-1 architecture is selected. The selection is based upon the guidelines given in [1].

\section{The Modified SA and Results}

For the simulation purpose of the MRAC the Buck converter is reflected as a system /plant. A Step down DC to DC is used in this Buck convertor which is inherently nonlinear due to switching operation. The specifications used for the design of Buck converter are as mentioned below: 
Switching frequency: $30 \mathrm{KHz}$

Inductor

Diode

Mosfet

: IRF 840

Capacitor

$: 47 \mu \mathrm{F}, 35 \mathrm{~V}$

Output Voltage

Input Voltage $\quad: 15 \mathrm{v}$ DC

The corresponding transfer function [2].

$G_{2}(s)=\left.\frac{\tilde{u}_{o}(s)}{\tilde{\delta}(s)}\right|_{\tilde{u}_{d}=0}=\frac{u_{d}}{a s^{2}+b s+c}$

Where $\quad a=L C \quad c=1+\frac{R_{L}}{R} \quad b=\frac{L}{R}+R_{L} C$

The transfer function of the buck converter

$G_{p}(s)=\frac{1.557 \times 10^{4}}{s^{3}+1.076 \times 10^{4} s+1.168 \times 10^{7}}$

The Tustin method is used to develop a discrete time version of (4) having the sampling frequency $30 \mathrm{KHz}$. The attained model equation is

$\mathrm{y}(\mathrm{n})=1.688 * \mathrm{y}(\mathrm{n}-1)-0.6986 * \mathrm{y}(\mathrm{n}-2)+0.0769 * \mathrm{u}(\mathrm{n}-1)+0.06828 * \mathrm{u}(\mathrm{n}-2)$

A second order stable system offering a $0.15 \mathrm{~ms}$ rise is obtained and settling time is $0.3 \mathrm{~ms}$. the reference model for discrete time is given as

$\mathrm{y}_{\mathrm{m}}(\mathrm{n})=1.2913 * \mathrm{y}_{\mathrm{m}}(\mathrm{n}-1)-0.2917 * \mathrm{y}_{\mathrm{m}}(\mathrm{n}-2)+0.2083 * \mathrm{r}_{\mathrm{m}}(\mathrm{n}-1)+0.1388 * \mathrm{r}_{\mathrm{m}}(\mathrm{n}-2)$

The result of the direct implementation of the algorithm in Table 1 is shown in Fig 3 (a). From the yield and the locus signal we can conclude that, if we choose the SA for optimizing the entire weight matrix of the Neural Net, there will be a small steady state error. Fig 3(b) represents the optimization algorithm applied only to the input to hidden weight vector.

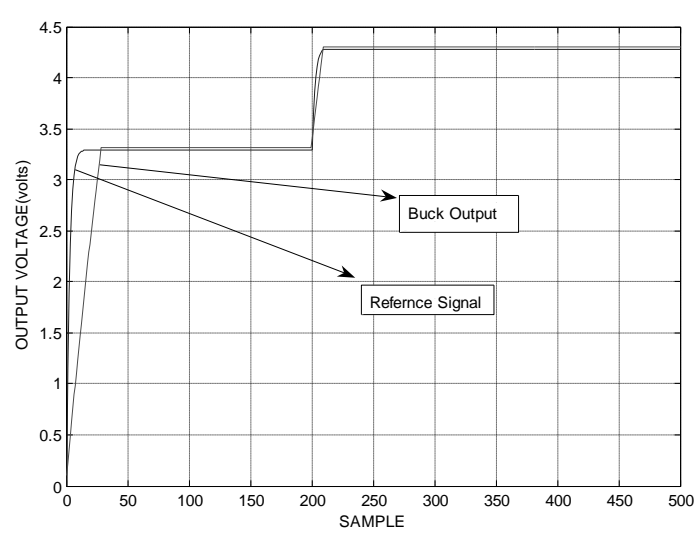

(a)

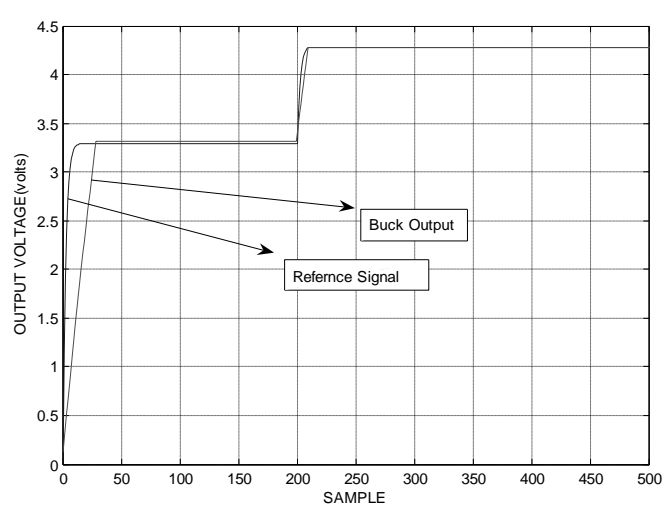

(b) 


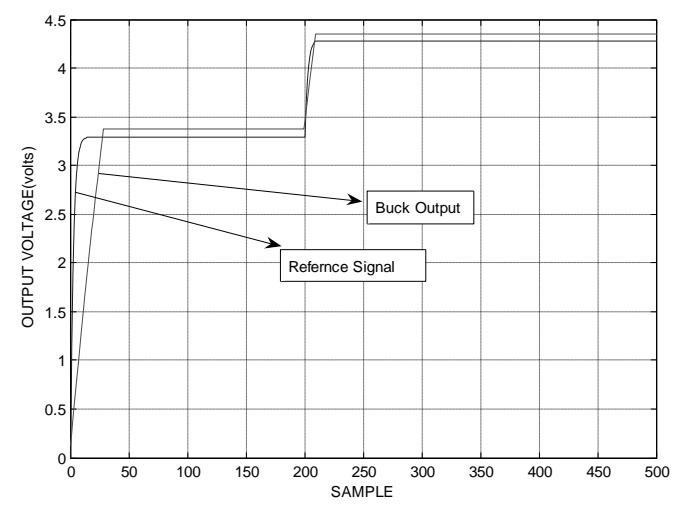

(c)

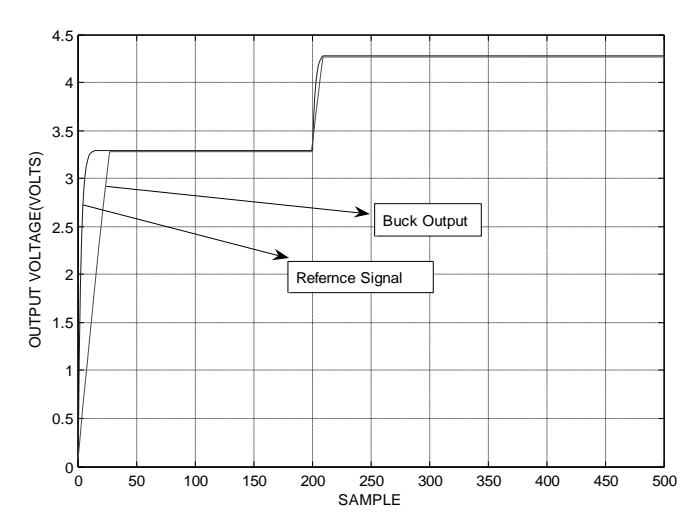

(d)

Fig 3: Startup response and response to reference change for buck converter (a) Response with conventional SA applied to $\mathrm{w}_{\mathrm{ij}}$, $\mathrm{w}_{\mathrm{jk}}$ weight vectors (b) Response with SA applied $\mathrm{w}_{\mathrm{ij}}$ weight vector (c) Response with SA applied only to $\mathrm{w}_{\mathrm{jk}}$ weight vector (d) Response with Modified SA

Now there is a improved reference change response but the startup response is defoliated. Also in Fig 3(c), if the optimization algorithm is applied to only hidden to output weight vectors, the result is a large steady state error. From the inspection of the above results we conclude that if for a single pass of algorithm, if we update only one set of weight vectors that is either input to hidden or hidden to output weights, we can arrive at good results with no exceed and no constant state error. The result of this modification are shown in Fig 3(d). One point to be noted here that in all the results shown we see the rise time of the system is constant.

To improve the rise time we have to control the temperature updating law.

$\mathrm{T}=\mathrm{T} * \mathrm{C}$

for the current application $\mathrm{C}=0.001$ and $\mathrm{T}=4000$;

With these modifications we can now reformulate the Table 1 as, shown in Table 2. If we see the modified algorithm, the overall processing time has been increased but the rise time of the system is not affected also system response is improved. 


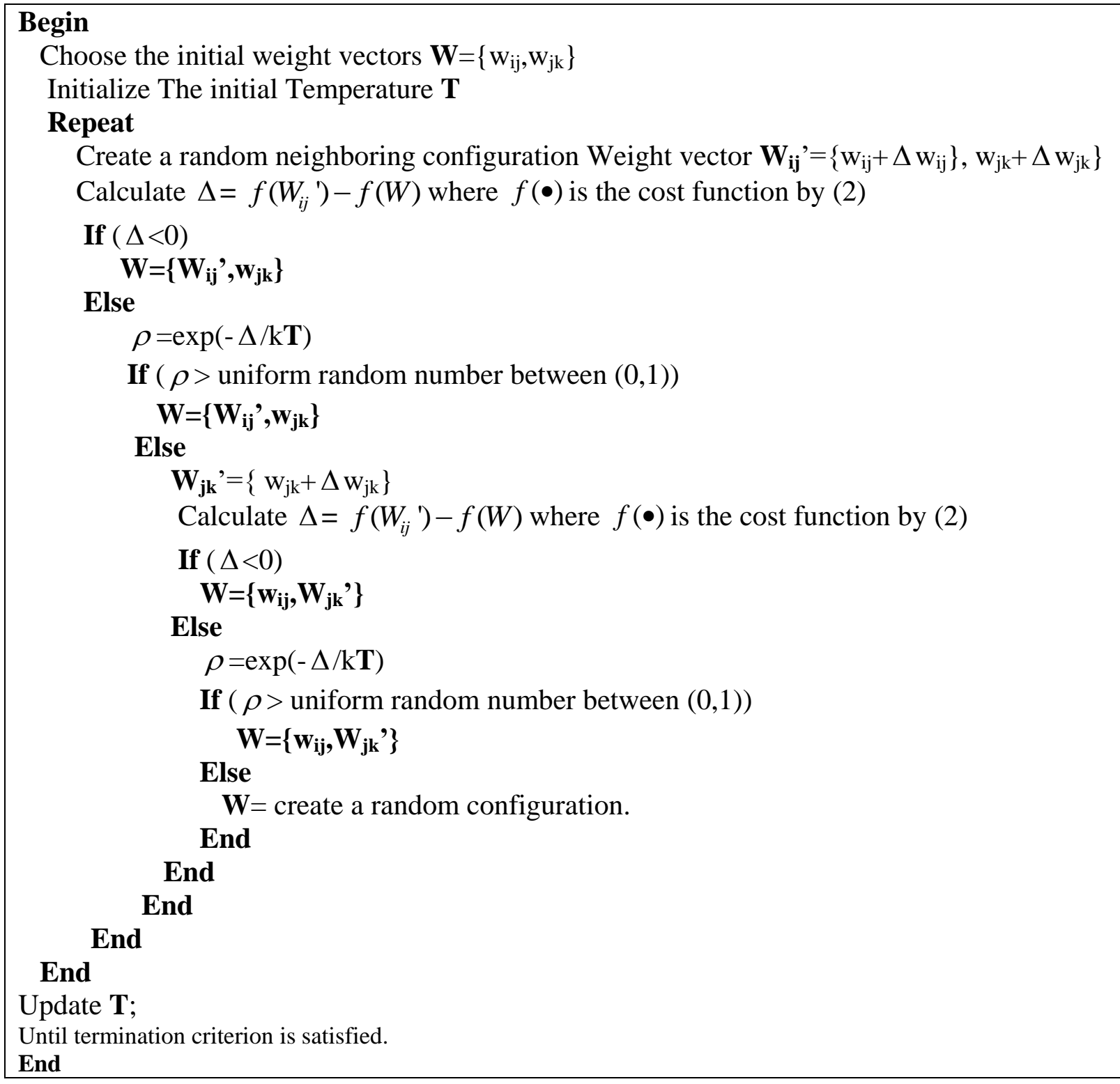

Table 2: The Modified SA algorithm for the Feed Forward Neural Network MRAC

\section{Conclusion}

General back propagation frame work is proposed with simulated annealing algorithm. This modified simulated annealing algorithm is simulated in Matlab and the improvements in simulated results are shown for buck converters. Similar principles can be applied to group of nonlinear plants /systems with varying structural certaininty. 


\section{REFERENCES}

[1] Cheng Xiang, Shenqiang Q. Ding, Tong Heng Lee "Geometrical Interpretation and Architecture selection of MLP" In Proc. IEEE Transaction on Neural Networks, Vol 16,No.1, January 2005.

[2] Dusan Gleich, Mira Milanovic, Suzana Uran, Franic Mihalic" Digitally controlled buck converter" In Proc. IEEE power electronics society; ISCAS- 2004 : V944-V947.

[3] Maria M. Goire, Jose M. Flores, Moises Bonilla and Lerohan S Baruch "Model Reference Neural control for a variable structure system by output feedback" 0-7803-7044-9/01/\$10.00 (C)2001 IEEE.

[4] H.D. Patino and Derong Liu "Neural Network-Based Model Reference Adaptive control system" In Proc. IEEE transaction on systems, Man and Cybernetics-Part B: Cybernetics, Vol. 30, No 1. February 2000

[5] M. Yuan, A. N. Poo and G. S. Hong "Direct neural control system: Nonlinear extention of adaptive control” In Proc. IEE Proc,-Control Theory Appl, Vol 142, No. 6. November 1995.

[6] Suwat Kuntanapreeda, Robert W. Gundersen, and R. Rees fullmer "Neural Network Model Reference Control Of Nonlinear Systems” 0-7803-0559-0/92 \$3.00 ( 1992 IEEE.

[7] Oxtega R. and Tang Yu "Robustness of Adaptive controllers- a survey" Automatica, vol 25, No. 5, PP. 651-667, 1989.

[8] Young-Moon Park, Myeon-Song Choi and Kwang Y. Lee "An optimal Tracking NeuroController for Nonlinear Dynamic Systems" IEEE Transaction on Neural Networks, Vol 7, No 5. September 1996.

[9] M. N. Gurcan, B. Sahiner, Heang-Ping Chan, L. Hadjiiski, and N. Petrick "Optimal Selection of Neural Network Architecture for CAD using Simulated Annealing” Proceedings of $22^{\text {nd }}$ Annual EMBS International Conference, July 23-28, 2000, Chicago IL.

[10] Lipo Wang and Kate Smith "On Chaotic Simulated Annealing" In Proc. IEEE Transection on Neural Networks”, Vol 9, No. 4, July 1998.

[11] Zhang, W., Maleki, A., Rosen, M.A. and Liu, J., 2018. Optimization with a simulated annealing algorithm of a hybrid system for renewable energy including battery and hydrogen storage. Energy, 163, pp.191-207.

[12] S.P.Gawande,Nilesh A Kubde, Manish S. Joshi, B.S.Sudame."Reactive power compensation of wind energy distribution system using Distributed Static Compensator". In Proc.IEEE $5^{\text {th }}$ International conference on Power Electronics, PP 1-5, December 2012.

[13] S.P.Gawande, B.S.Sudame, R.N.Nagpure, Jawed A.Sheikh and B.Y.Bagde,"Comprehensive Analysis of Voltage Sag and its Mitigation using Multilevel Dynamic Voltage Restorer." In Proc. Journal of Engineering and Applied Science, Vol 04(01), PP 20-25, January 2019. 